\title{
Presentation of Intrahepatic Cholangiocarcinoma with Fulminant Hepatic Failure: A Case Report
} \author{
Pradeep G. Mathew ${ }^{4}$ Rithu Sebastian ${ }^{4}$ \\ ${ }^{1}$ Department of Radiology, VPS Lakeshore Hospital, Kochi, Kerala, \\ India \\ 2Department of Internal Medicine and Rheumatology, VPS \\ Lakeshore Hospital, Kochi, Kerala, India \\ ${ }^{3}$ Department of Pathology, VPS Lakeshore Hospital, Kochi, Kerala, \\ India \\ ${ }^{4}$ Department of Gastroenterology, VPS Lakeshore Hospital, Kochi, \\ Kerala, India
}

Thara Pratap ${ }^{1}$ Muhammed Jasim Abdul Jalal ${ }^{2}$ Pushpa Mahadevan ${ }^{3}$

\begin{abstract}
Address for correspondence Muhammed Jasim Abdul Jalal, MBBS, DNB (Family Medicine), MNAMS, MRCGP(UK), MRCP(UK), Department of Internal Medicine and Rheumatology, VPS Lakeshore Hospital, Nettoor P.O, Kochi 682040, Kerala, India (e-mail: jasimabduljalal@yahoo.com).
\end{abstract}
Abstract
Keywords
- fulminant hepatic failure
- intrahepatic cholangiocarcinoma
- transjugular liver biopsy

Fulminant hepatic failure as initial presentation due to diffuse parenchymal infiltration by cholangiocarcinoma is a rare entity. We present the case of a 49-year-old female patient who had a fatal outcome with acute liver failure due to diffuse intrahepatic cholangiocarcinoma. No definite mass lesion was identified on cross-sectional imaging. The final diagnosis was made on transjugular liver biopsy. This discussion highlights the possibility of infiltrative cholangiocarcinoma as a rare cause of fulminant hepatic failure.

\section{Introduction}

Intrahepatic cholangiocarcinoma (ICC) is the second most common malignant tumor of the liver. It constitutes $10 \%$ of cholangiocarcinomas. ${ }^{1}$ By definition, intrahepatic cholangiocarcinoma originates proximal to second degree bile ducts This tumor is classified on the basis of gross morphological features into mass forming, periductal infiltrating, and intraductal type. Cholangiocarcinoma often presents at an advanced stage and most of the cases are unresectable. The disease often has a fatal outcome. The prevalence of this disease is highest in Southeast Asian countries such as Thailand and is less in the Western world. In Asian countries, parasites such as liver fluke-Clonorchis sinensis and Opisthorchis viverrini-and hepatolithiasis are major risk factors, other causes being biliary tract disease such as primary sclerosing cholangitis, primary biliary cirrhosis, choledochal cysts, infective causes such as hepatitis B and C virus, alcoholic liver disease, and smoking, though most of the cases are idiopathic. The disease is more common in males than females and occurs most frequently between sixth and seventh decade.
The incidence of the disease is on the rise in the west ${ }^{2}$ and in younger population.

\section{Case Presentation}

A 49-year-old lady presented with recurrent dull aching pain in the right upper abdomen and jaundice of 1 month duration. It was associated with dyspnea on exertion. On examination, she was icteric and had abdominal distension with shifting dullness, bilateral pedal edema, and facial puffiness. There was no history of pruritus, clay colored stools, weight loss, fever, or prodromal symptoms. She was on ayurvedic medications for urinary tract infection 3 months back. Her liver function (AST/ALT/ALP 92/43/331) was deranged and coagulation parameters (PT/INR/Index 15.2/1.13/90.8\%) were found to be normal. Viral markers were nonreactive. Ascitic fluid analysis was lymphocyte predominant with negative cytology. Ascitic fluid albumin was normal. Upper gastrointestinal endoscopy showed grade II esophageal varices and small fundic varix suggesting a diagnosis of decompensated liver disease. received

June 6, 2019

accepted after revision

June 16, 2019

published online

August 22, 2019
DOI https://doi.org/

10.1055/s-0039-1694800

ISSN 2581-9933.
(C)2019 Indian Society of

Gastrointestinal and Abdominal

Radiology
License terms

()(1) $\Theta$ 


\section{Cross Sectional Imaging}

Triple phase computed tomography (CT) scan was performed with GE 64 slice scanner light speed VCT XTE. Ninety milliliter of intravenous contrast Ultravist $350 \mathrm{mg}$ was given at a flow rate of $3.8 \mathrm{~mL} / \mathrm{min}$. CT images were obtained with bolus tracking technique with a scan delay of 4 to 5 seconds after a set contrast threshold of $100 \mathrm{HU}$. Arterial, porto-venous, hepatic venous and delayed scans were taken. The images were viewed on a dedicated GE workstation (AW 4.4) and coronal, sagittal reconstructions were also analyzed.

Contrast-enhanced computed tomography (CECT) scans showed hepatomegaly with patchy ill-defined hypo-dense areas involving both lobes better appreciated in portal and venous phase scans (-Figs. 1-4) than in arterial phase. No definite enhancing mass lesions, ductal thickening, or biliary
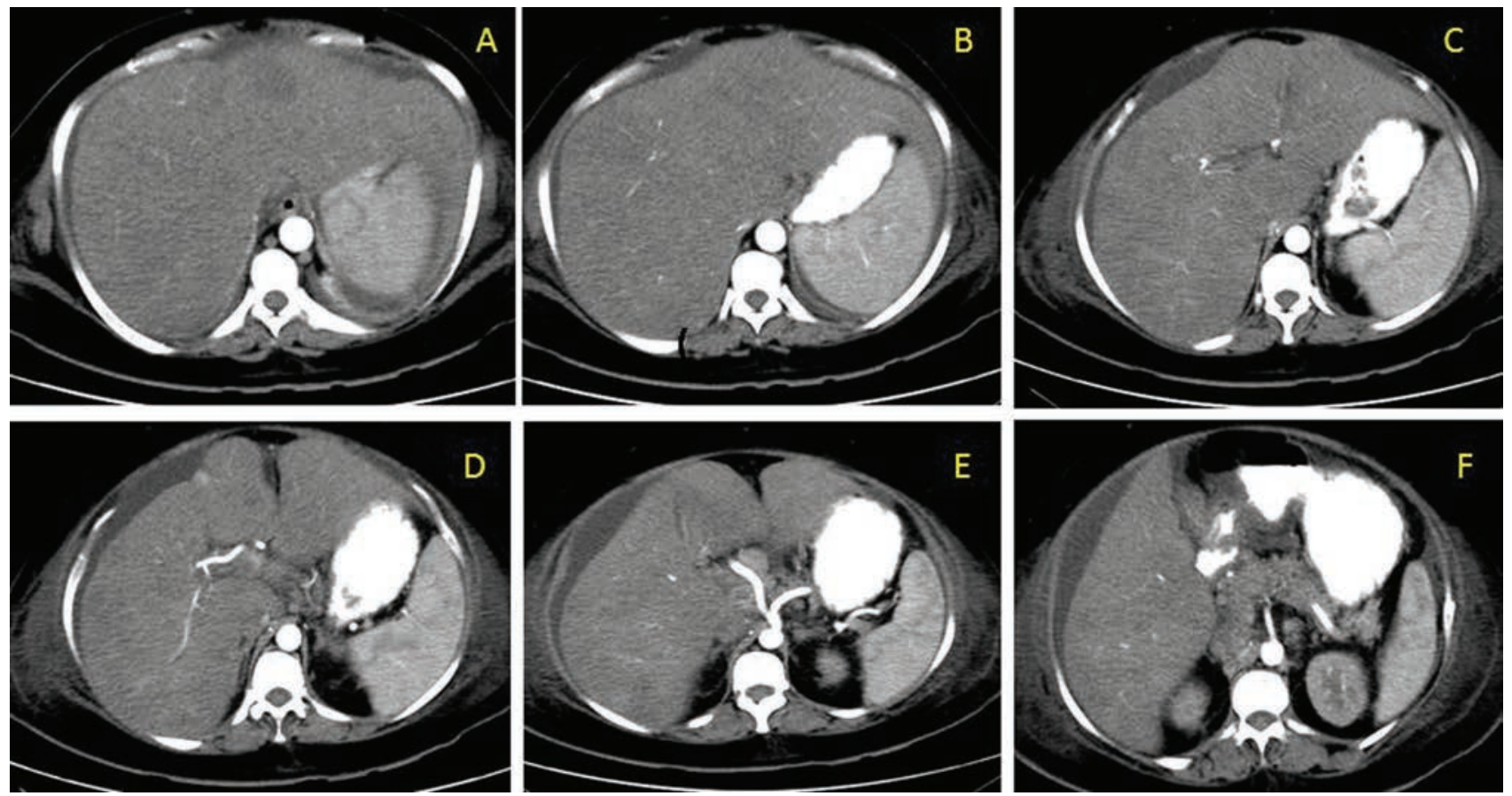

Fig. 1 (A-F, arterial phase): 64 slice contrast CT scans showing hepatomegaly with lobulated contour of liver with patchy hypo-dense areas involving both lobes of liver. CT, computed tomography.
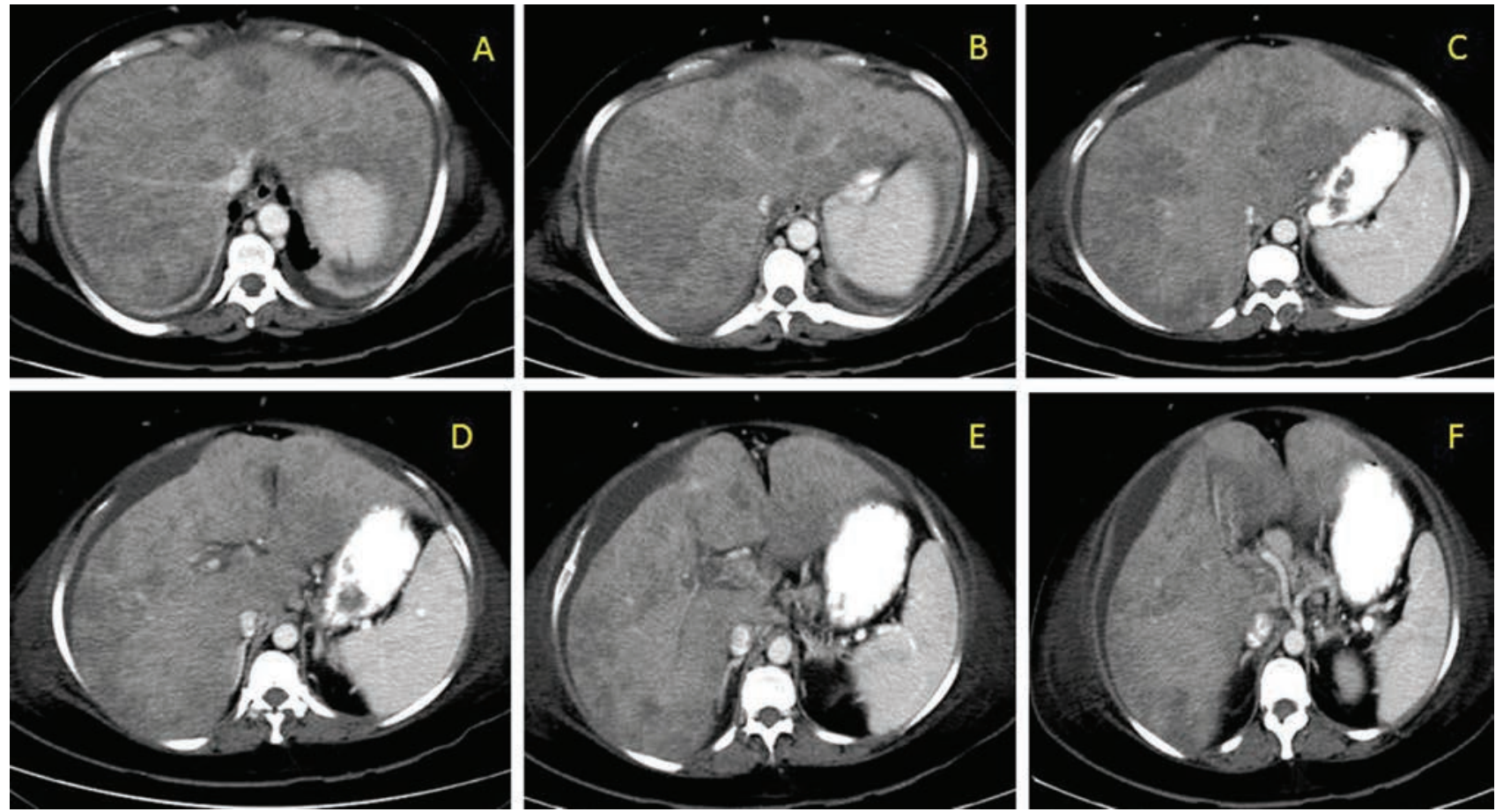

Fig. 2 (A-F, portal venous phase): contrast CT scans showing hepatomegaly with multiple patchy geographic hypo-dense areas involving both lobes of liver, better appreciated than in arterial phase scans. CT, computed tomography. 
dilatation was noted. There was associated small segment portal vein thrombosis involving right posterior segmental branch. Rest of the portal vein branches was normal. In addition, there were minimal ascites and multiple small retroperitoneal nodes. Differential diagnosis considered was chronic liver disease, infiltrative liver disease, and metastatic liver disease. However, there was no evidence of primary in the chest or abdomen.

In view of acute liver failure of unknown etiology, transjugular liver biopsy was performed. Histopathology revealed the diagnosis of infiltrating periductal adenocarcinoma with immunohistochemistry confirming the diagnosis of primary cholangiocarcinoma liver. Oncology evaluation was sought, but she deteriorated rapidly.

\section{Histopathology}

The tumor was of periductal type with multiple foci of infiltrating adenocarcinoma predominantly of clear cell type (-Figs. 5 and $\mathbf{6}$ ). Clear cell changes have been uncommonly reported in patients with cholangiocarcinoma.
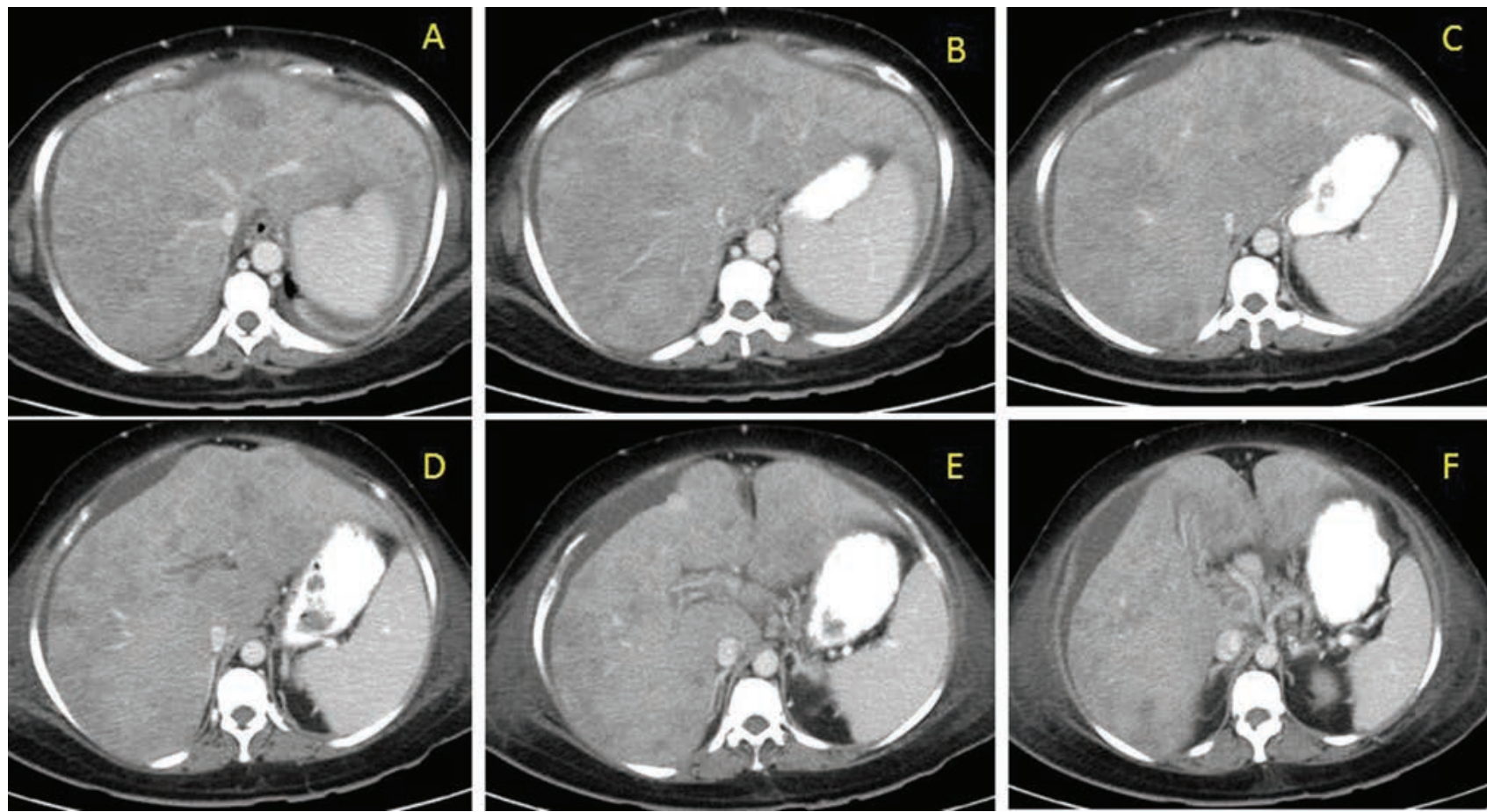

Fig. 3 (A-F, hepatic venous phase): contrast CT scans show hepatomegaly with patchy hypo-dense areas involving both lobes. (D) Shows smal segment portal venous thrombosis. CT, computed tomography.
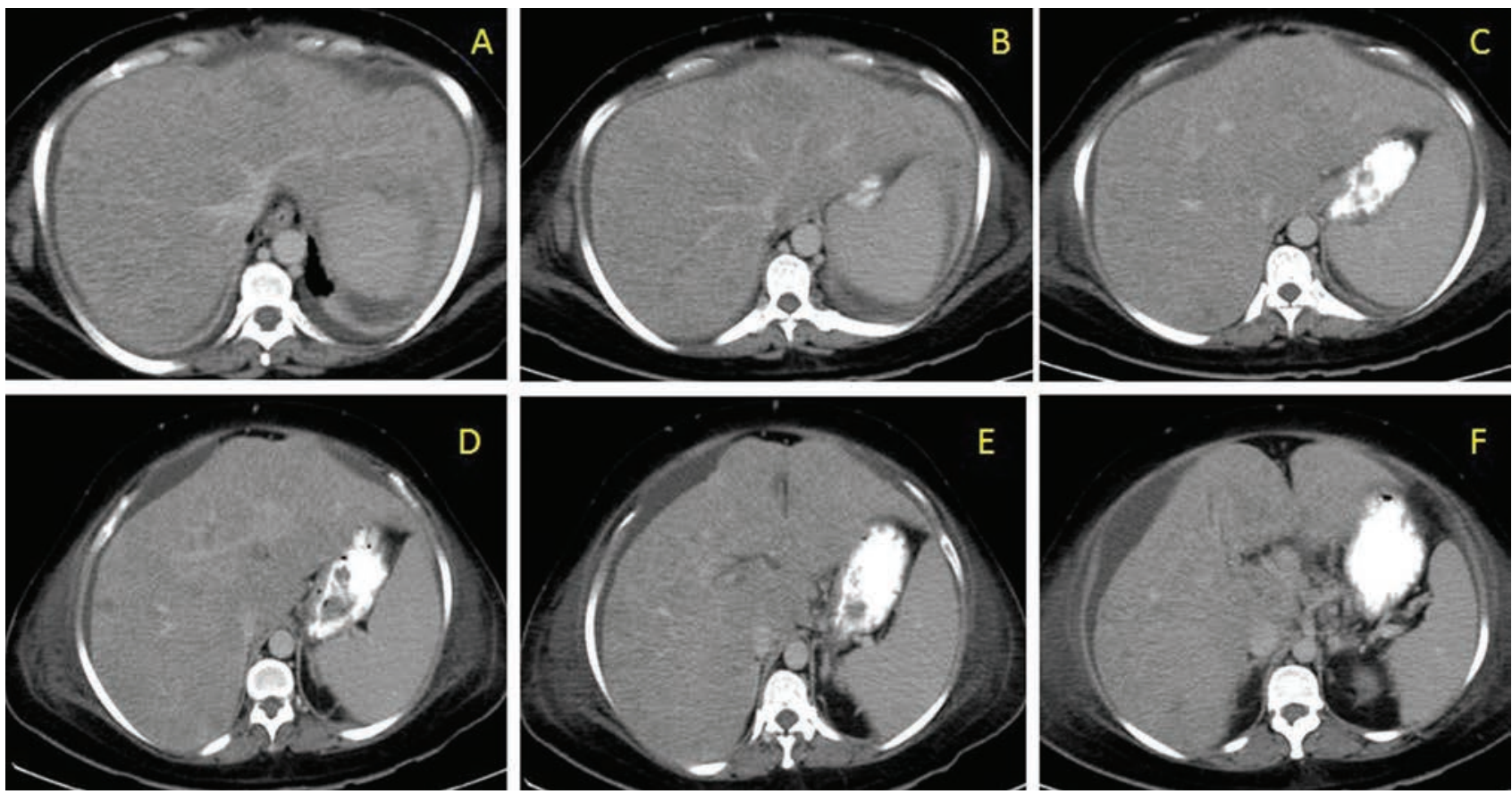

Fig. 4 (A-F, delayed scans): further iso-dense appearance of lesions is shown. 


\section{multiple foci of infiltrating adenocarcinoma}

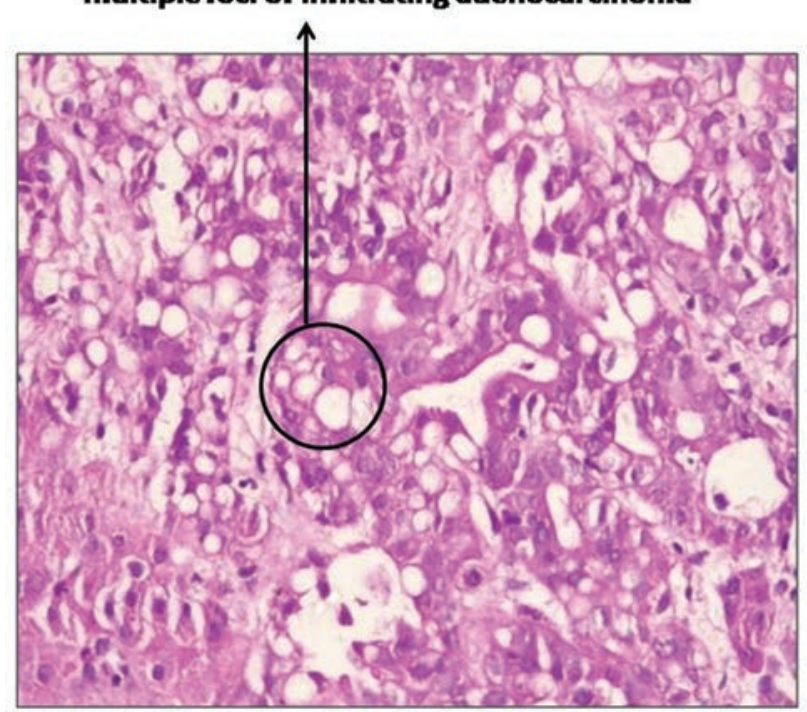

Fig. $5 \mathrm{H} \& \mathrm{E}$ staining, high power view shows peri-ductal infiltrating type of cholangiocarcinoma with multiple foci of infiltrating adenocarcinoma. H\&E, hematoxylin and eosin.

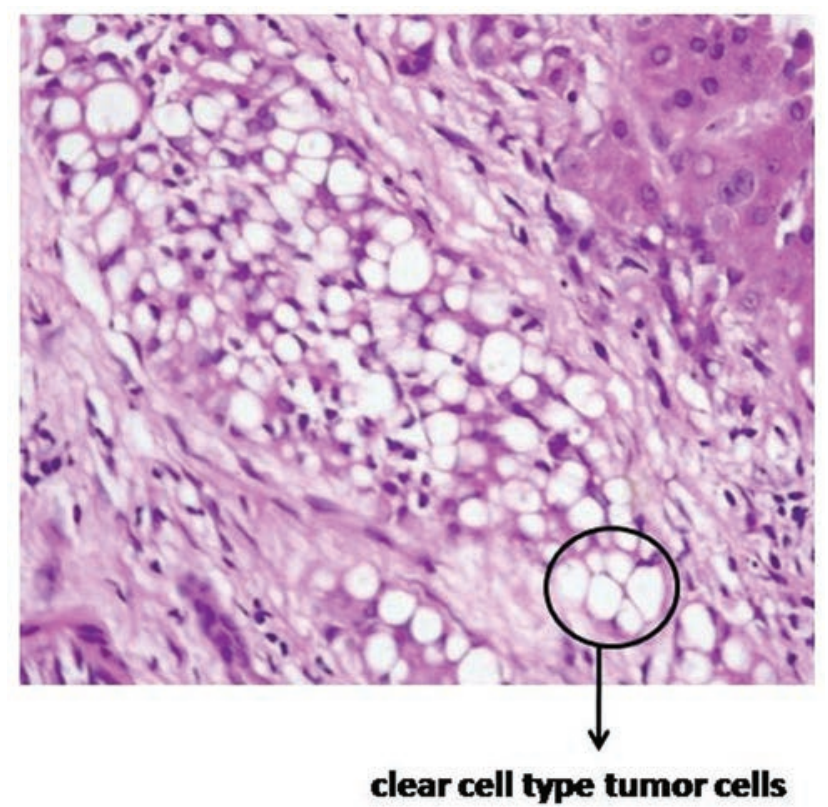

Fig. 6 H\&E staining, high power view showing clear cell type tumor cells. H\&E, hematoxylin and eosin.

Immunohistochemistry showed tumor cells positive for cytokeratin 7 (CK7) and negative for hepatocyte antibody (-Figs. 7 and 8 ).

\section{Discussion}

ICC with typical imaging findings can be diagnosed easily. Our patient presented with hepatomegaly with patchy ill-defined hypo-dense areas and no definite mass on cross sectional imaging. In spite of periductal infiltrating type on histopathology, there was no biliary duct thickening or bile duct obstruction. This is an extremely rare presentation on imaging.

Pathology showed extensive periductal type of disease explaining the cause of acute liver failure, which is often

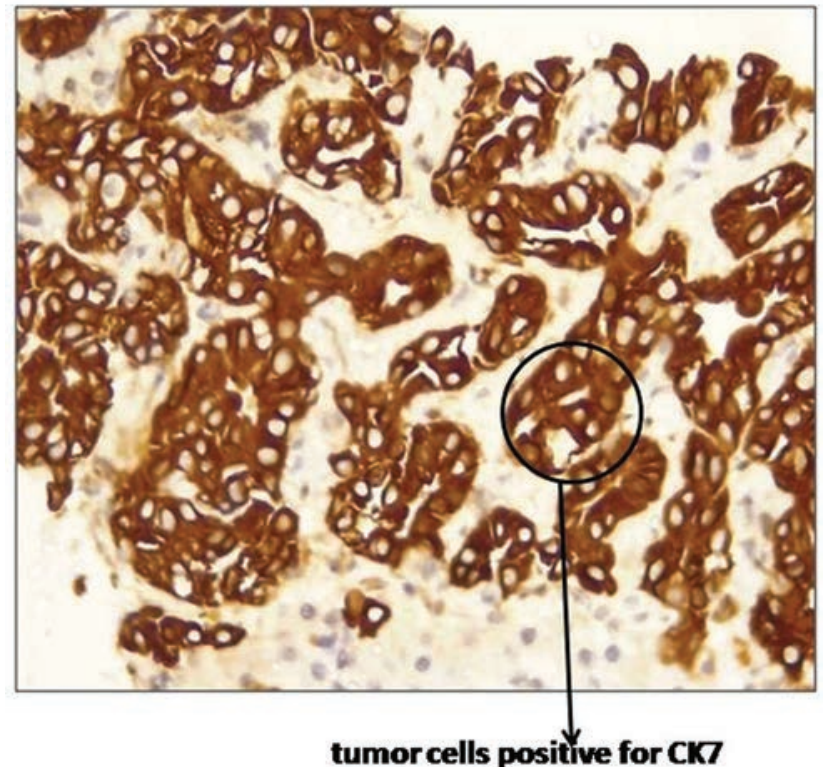

Fig. 7 Immunohistochemistry showing tumor cells positive for CK7. CK7, cytokeratin 7.

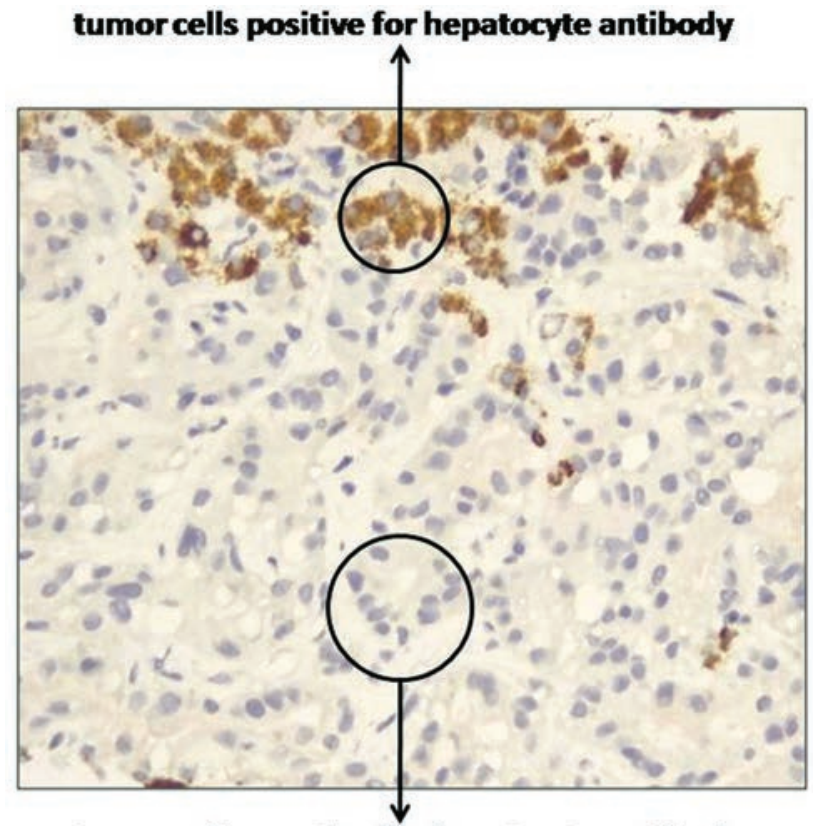

tumor cells negative for hepatocyte antibody

Fig. 8 Immunohistochemistry showing tumor cells negative for hepatocyte antibody.

due to neoplastic infiltration of hepatic sinusoids leading to parenchymal infarction and secondary necrosis of hepatocytes. ${ }^{3}$ Replacement of 80 to $90 \%$ of hepatic parenchyma by neoplastic cells could lead to jaundice and liver failure. ${ }^{4,5}$

Literature search reveals only few cases of primary cholangiocarcinoma with acute liver failure. The diagnosis in these cases was made on liver biopsy or autopsy. ${ }^{6}$ In none of the cases, preoperative diagnosis could not be made as no definite masses could be identified on CT scan.

Fulminant hepatic failure due to primary liver tumors other than hepatocellular carcinoma is very rare. There are few case reports of acute liver failure due to primary hepatic 
angiosarcoma. ${ }^{7,8}$ Infiltrative deposits due to hematopoietic diseases and metastatic deposits from primary breast and lung cancers ${ }^{3}$ are already known entities.

\section{Conclusion}

Cholangiocarcinoma presenting as diffuse infiltrating intrahepatic disease with no definite mass lesions on $\mathrm{CT}$ is very rare. The role of liver biopsy is crucial to make the diagnosis given the atypical imaging findings and presentation. This alerts us to the rare possibility of primary diffuse infiltrating cholangiocarcinoma as a cause of fulminant hepatic failure.

\section{Conflict of Interest}

None declared.

\section{References}

1 Buettner S, van Vugt JL, IJzermans JN, Groot Koerkamp B. Intrahepatic cholangiocarcinoma: current perspectives. OncoTargets Ther 2017;10:1131-1142
2 Saha SK, Zhu AX, Fuchs CS, Brooks GA. Forty-year trends in cholangiocarcinoma incidence in the U.S.: intrahepatic disease on the rise. Oncologist 2016;21(5):594-599

3 Athanasakis E, Mouloudi E, Prinianakis G, Kostaki M, Tzardi M, Georgopoulos D. Metastatic liver disease and fulminant hepatic failure: presentation of a case and review of the literature. Eur J Gastroenterol Hepatol 2003;15(11):1235-1240

4 Nazario HE, Lepe R, Trotter JF. Metastatic breast cancer presenting as acute liver failure. Gastroenterol Hepatol (N Y) 2011;7(1):65-66

5 Hanamornroongruang S, Sangchay N. Acute liver failure associated with diffuse liver infiltration by metastatic breast carcinoma: a case report. Oncol Lett 2013;5(4):1250-1252

6 Vakil A, Guru P, Reddy DR, Iyer V. Diffuse cholangiocarcinoma presenting with hepatic failure and extensive portal and mesenteric vein thrombosis. BMJ Case Rep. 2015; 2015:bcr2014209171

7 Bhati CS, Bhatt AN, Starkey G, Hubscher SG, Bramhall SR. Acute liver failure due to primary angiosarcoma: a case report and review of literature. World J Surg Oncol 2008;6:104

8 López R, Castro-Villabón D, Álvarez J, Vera A, Andrade R. Hepatic angiosarcoma presenting as acute liver failure in young adults. Report of two cases and review of literature. Case Reports in Clinical Medicine 2013;2:439-444 